\title{
Boning up on dinosaurs
}

\author{
The Microstructure of Dinosaur Bone: \\ Deciphering Biology with Fine-Scale \\ Techniques \\ by Anusuya Chinsamy-Turan \\ Johns Hopkins University Press: 2005. \\ 216 pp. $\$ 85, £ 56.50$
}

\section{Luis M. Chiappe}

Studies of the microstructure of dinosaur bone began soon after the first scientific reports of these Mesozoic behemoths, but it took nearly 150 years for this discipline to enter the mainstream of dinosaur palaeontology. Nowadays, these studies are used to infer numerous aspects of dinosaur biology, from growth rates and physiological strategies to skeletal biomechanics and developmental patterns, and are even used as a source of new characters for phylogenetic inference. The Microstructure of Dinosaur Bone by Anusuya Chinsamy-Turan is the furst book-length review of this rapidly advancing line of palaeontological research.

Chinsamy-Turan begins by introducing a host of topics, including the basics of bone composition, histological studies, the changes that occur on death and fossilization, and dinosaur evolution and ecology. Next, she examines the biological meaning of bone microstructure. She looks at bone-tissue dynamics (the rate at which it is deposited, and whether it occurs without interruption or with growth rings) and vascularization (the number of blood vessels), and explains how they can provide clues to the ontogenetic, biomechanical and ecological contexts of bone.

After that she turns her attention to dinosaurs, with a brief history of dinosaur-bone histology and a review of the diverse tissues found in their bones. We then reach the heart of the book, with a discussion of how microstructural studies of fossil bone inform our understanding of the growth patterns of extinct vertebrates. Particularly useful is the author's emphasis on the caveats for inferring some aspects of growth dynamics from fossil bones. Then she covers a number of studies, mostly her own, on the bone histology of premodern, Mesozoic birds - dinosaurs' most immediate descendants - and the important changes in growth patterns thought to have occurred during their evolution. Finally, she delves into dinosaur physiology to see if bone microstructure can tell us whether some, all or none of these animals were warm-blooded.

The book draws on Chinsamy-Turan's extensive studies of the bone microstructure of living tetrapods. It is perhaps her deep understanding of the complexity of skeletal growth that leads her continuously to warn against making a strong connection between the different types of tissue preserved in fossilized bones and either specific rates of bone formation or dinosaur physiology. Throughout the book, Chinsamy-Turan is resolute in defending her views from opposing perspectives; at times it feels like we are watching a television commercial for a video-game. Fortunately, however, the different contenders in the modern arena of dinosaur-bone microstructure agree on several fundamentals. Thanks to work by Chinsamy-Turan and her colleagues and mentors, we know that even if the growth strategies of dinosaurs varied greatly, these animals grew at rates substantially faster than extant nonavian reptiles (even the largest dinosaurs reached adult size in less than three decades). We have also learned that the same rate of bone formation may lead to the creation of different types of bone tissue, and that growth series and data from different skeletal elements are critical for confidently inferring the growth patterns of extinct dinosaurs.

Not surprisingly, the area of greatest disagreement is the largely conjectural issue of whether, in a physiological sense, dinosaurs more closely resembled cold-blooded reptiles or warm-blooded birds and mammals. Most researchers agree that rapid growth cannot be confidently correlated with warm-bloodedness, and most of the disagreement seems to be akin to whether a glass is viewed as halffull or half empty. Chinsamy-Turan highlights how the bone microstructure of dinosaurs shows features that are either frequently found in non-avian reptiles or widespread among birds and mammals, but she wisely advises us not to draw definitive physiological conclusions from bone microstructure.

Chinsamy-Turan is at her best when she discusses the microstructure of bone and its

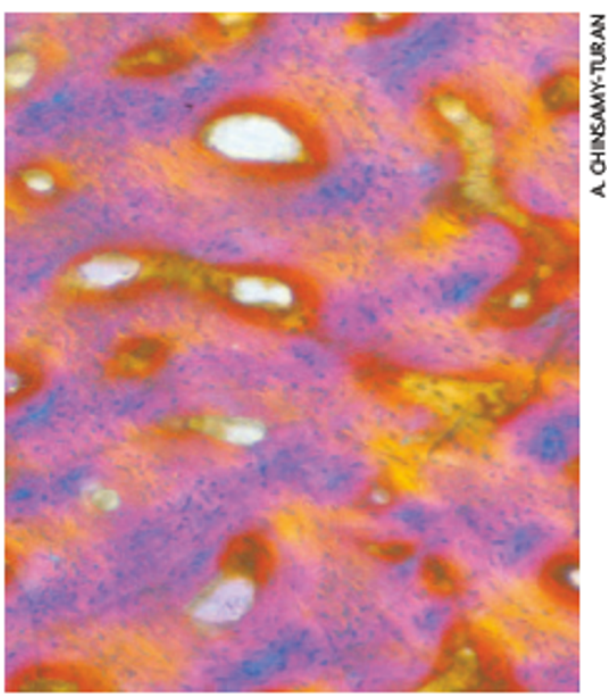

Growth area:microstructure study reveals stages of bone formation in the dinosaur Dryosaurus.

significance for understanding the biology of fossil vertebrates. However, her condusions about the linkage between the origin of warmbloodedness and the divergence of ornithurines (the group induding Hesperornis, Ichthyornis and all living birds) are weakened by the endorsement of views that have a morphologi$\mathrm{cal}$ and functional basis that is not clearly substantiated. Despite these shortcomings, Chinsamy-Turan's book is a tour de force that gives readers an updated synopsis of a fastgrowing field of vertebrate palaeontology. The book is a must-read for anybody interested in the biology of one of the most fascinating animals in the history of our planet.

Luis M. Chiappe is at the Dinosaur Institute, Natural History Museum of Los Angeles County, 900 Exposition Boulevard, Los Angeles, California 90007, USA.

\section{Summing up physics}

\section{The Equations: Icons of Knowledge by Sander Bais \\ Harvard University Press: 2005. 96 pp. \\ \$18.95, £11.95, €17.50}

\section{Malcolm Longair}

Perhaps the most common comment I receive after delivering a public lecture is: "I enjoyed the lecture, but I couldn't understand the maths." This difficulty doesn't just affect the public but is one that students and scientists encounter throughout their careers: how does one connect the behaviour of the real world with the mathematics used to describe it? After years of practice and hard thinking, professionals become adept at making this connection, which I regard as perhaps the greatest challenge in teaching mathematical physics.

Sander Bais's little book The Equations tackles this problem from the point of view of 17 of the basic sets of physics equations, which the subtitle refers to as "icons of knowledge ${ }^{x}$. In a book of only 96 pages, it is a real challenge to do much more than indicate to the reader the enormous richness of these equations and the imaginative ways they can be used to extend our understanding of the workings of nature at all levels. The equations are presented in their final definitive forms without any discussion of how they came about or why they have these forms. This latter aspect is not part of Bais's agenda, which is a pity. Still, if it were, the book would have grown out of all proportion.

In the first few pages there is a lightning review of elementary mathematical operations, and this helps the reader understand the simpler equations, such as Newton's laws of motion and the continuity equation. It is debatable, however, whether these insights enable the reader to understand the importance of 